\title{
Actividades preventivas e indicadores - Quanto tempo sobra?
}

Daniel Pinto*, Susana Corte-Real**, José Mendes Nunes***

\section{RESUMO}

Objectivos: Determinar o número de consultas necessário durante um ano para realizar alguns procedimentos preventivos seleccionados e, nas pessoas com diabetes ou hipertensão arterial, cumprir os indicadores de desempenho contratualizados.

Métodos: Estudo descritivo baseado na lista de utentes de um médico de família (1587 utentes). Considerou-se a vigilância de crianças e jovens, grávidas, diabéticos e hipertensos e os rastreios dos cancros da mama, do colo do útero e colo-rectal, da hipertensão arterial, da dislipidémia e da obesidade. Foi definido o seguimento adequado para cada situação com base em normas de orientação clínica, na taxa de cobertura contratualizada pela Unidade de Saúde Familiar e na definição de indicadores de desempenho da Missão para os Cuidados de Saúde Primários. Considerou-se a realização de cada actividade em separado ou de múltiplas actividades preventivas e de vigilância na mesma consulta.

Resultados: Seriam necessárias 2848,5 consultas para levar a cabo as actividades preventivas e de vigilância estudadas se estas fossem realizadas em separado. Considerando a possibilidade de efectuar múltiplas actividades na mesma consulta, seriam necessárias 2008,9 consultas por ano (50,6\% do total realizado no ano anterior).

Conclusões: A realização das actividades preventivas e o cumprimento de indicadores de desempenho exigem um dispêndio considerável de tempo. Antes de serem atribuídas mais tarefas aos médicos de família, será necessário estudar o impacto de cada uma em termos recursos a despender para a sua execução.

Palavras-chave: Medicina Familiar; Recursos de Saúde; Incentivo de Desempenho; Prevenção Primária.

\section{INTRODUÇÃO}

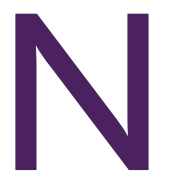

os últimos anos a promoção da saúde e a prevenção da doença têm adquirido um lugar de destaque na prática médica, em particular, na medicina geral e familiar. ${ }^{1}$ Porém, alguns autores têm posto em causa o peso relativo das actividades preventivas na consulta do médico de família. Argumentam que as fronteiras do que é considerado prevenção têm vindo a ser sucessivamente alargadas sem que isso se traduza em ganhos em saúde. ${ }^{2,3}$ Em outras ocasiões, factores de risco são en-

\footnotetext{
* Assistente de Medicina Geral e Familiar. Assistente convidado voluntário do Departamento de Medicina Geral e Familiar da Faculdade de Ciências Médicas da Universidade Nova de Lisboa. USF São Julião - Centro de Saúde de Oeiras

** Assistente de Medicina Geral e Familiar. USF São Julião - Centro de Saúde de Oeiras

*** Chefe de Serviço de Medicina Geral e Familiar. Assistente convidado do Departamento de Medicina Geral e Familiar da Faculdade de Ciências Médicas da Universidade Nova de Lisboa. UCSP de Almeirim - ACES da Lezíria
}

carados como «doenças» que requerem tratamento e acompanhamento por parte do médico. ${ }^{4}$ Esta ênfase na prevenção e controlo de factores de risco poderá desviar recursos (que são escassos) do tratamento dos verdadeiramente doentes para aqueles que são saudáveis, mais jovens e com maior poder económico. ${ }^{5} \mathrm{Ou}-$ tros autores argumentam que a responsabilidade da implementação de uma forma sistemática de medicina preventiva na comunidade não deve ser dos médicos de família, devendo estes limitar-se a oferecer actividades preventivas específicas em doentes de alto risco. $^{6}$

Em Portugal, tem-se procurado medir o desempenho do sistema de cuidados de saúde primários através da avaliação de um número restrito de actividades clínicas, argumentando que tal permitiria alcançar melhores resultados em saúde. ${ }^{7}$ Muitos destes indicadores de desempenho avaliam a execução de procedimentos 
preventivos (como o rastreio de certos cancros), controlo de factores de risco (como a hipertensão arterial) e seguimento de algumas doenças (como a diabetes). Os médicos de família podem optar por um vencimento dependente do cumprimento dos indicadores de desempenho contratualizados. ${ }^{9}$

Contudo, existe alguma evidência de que a orientação do sistema de saúde para o pagamento associado ao desempenho poderá reduzir a qualidade dos cuidados não abrangidos por estes indicadores. ${ }^{10,11}$ Alguns autores argumentam que as diferenças medidas pelos indicadores de desempenho podem não se traduzir por alterações clinicamente significativas na saúde da população, uma vez que estes não medem parâmetros clinicamente relevantes, apenas processos que teoricamente levariam a melhores resultados. ${ }^{12}$ Por outro lado, corre-se o risco de os médicos passarem a dar prioridade ao cumprimento dos indicadores avaliados, descurando áreas ou utentes onde o seu investimento poderia ter um efeito clínico mais significativo. ${ }^{10,12}$

Nos Estados Unidos da América, foi estimado que um médico de cuidados primários com uma população de 2500 utentes necessitaria de 1773 horas por ano para cumprir todas as actividades preventivas recomendadas pela United States Preventive Services Task Force. ${ }^{13}$ Isto seria equivalente a 1064 horas numa lista de 1500 utentes. Um médico de família em Portugal, com um horário de 30 horas assistenciais por semana e que trabalhe 46 semanas por ano, cumpre 1380 horas de consulta.

Contudo, não existe, até ao momento, uma estimativa de quanto tempo poderá ser necessário aos médicos de família portugueses despender para cumprir as actividades preventivas e os indicadores de desempenho contratualizados e, dessa forma, saber até que ponto o seu cumprimento poderá interferir com as restantes actividades. É do nosso conhecimento o trabalho de A Fernandes e colaboradores, que procuraram estimar as necessidades de consultas em função da pirâmide etária da lista de utentes. ${ }^{14}$

O presente trabalho teve por objectivo determinar, na lista de um médico de família, o número de consultas necessário durante um ano para realizar alguns procedimentos preventivos seleccionados e, nas pessoas com diabetes ou hipertensão arterial (HTA), cumprir os indicadores de desempenho contratualizados.

\section{MÉTODOS}

Estudo descritivo transversal baseado na lista de utentes de um médico de família. A lista foi estudada pelos autores no âmbito do internato médico de medicina geral e familiar, construindo a lista de problemas para todos os utentes com base no processo clínico electrónico e em papel. Os problemas foram classificados utilizando a Classificação Internacional de Cuidados Primários - 2. ${ }^{a}$ edição (ICPC-2). ${ }^{15}$ No momento em que foi estudada (Dezembro 2008), a lista era composta por 1587 utentes, com uma idade média de 38,5 anos e $54,9 \%$ eram do sexo feminino.

Foram seleccionados alguns procedimentos preventivos com base no seu peso na actividade dos médicos de família, capacidade para identificar o número de consultas necessário para os realizar e evidência científica que suporta a sua execução. Seleccionaram-se a HTA, a diabetes, a vigilância de crianças e jovens e as grávidas por serem muito frequentes na consulta do médico de família e existirem orientações explícitas acerca do seguimento destes utentes.

O seguimento adequado foi definido através das orientações da Direcção-Geral de Saúde, ${ }^{16-18}$ da United States Preventive Services Task Force, ${ }^{19}$ do Royal Australian College of General Practitioner ${ }^{20}$ e do Núcleo de Actividades Preventivas da Associação Portuguesa dos Médicos de Clínica Geral. ${ }^{21}$ Foram tidas em conta as taxas de cobertura contratualizadas para o ano de 2009 pela Unidade de Saúde Familiar (USF) a que os utentes pertenciam.

Nas crianças e jovens considerou-se ser necessária uma consulta em cada idade-chave definida pela Direcção-Geral de Saúde: recém-nascido, um mês, dois meses, quatro meses, seis meses, nove meses, doze meses, quinze meses, dezoito meses, dois anos, três anos, quatro anos, cinco a seis anos, oito anos, onze a treze anos, quinze anos e dezoito anos. ${ }^{16}$

Para as grávidas de risco normal considerou-se que o seguimento adequado implicaria oito consultas ao longo da gravidez (em média, uma por mês), havendo referenciação para o hospital às 36 semanas. ${ }^{17}$ No caso das gravidezes de alto risco, considerou-se que seria apenas feita uma consulta para referenciação da grávida ao obstetra.

Nos diabéticos tipo 2 estabeleceu-se como mínimo necessário quatro consultas anuais (uma por trimestre), 
para permitir cumprir o indicador de registo de três valores de hemoglobina glicosilada. ${ }^{8}$ Para os diabéticos de outros tipos definiu-se como necessária apenas uma consulta por ano, uma vez que o seguimento seria feito nos cuidados secundários ou terciários.

Para os doentes hipertensos com ou sem complicações consideraram-se necessárias, no mínimo, duas consultas por ano para cumprir o indicador de registo da pressão arterial em cada semestre. ${ }^{8}$

Para rastreio do cancro da mama considerou-se necessária a realização de uma mamografia de dois em dois anos nas mulheres dos 50 aos 69 anos, inclusive (metade das mulheres em cada ano). ${ }^{20}$ Para rastreio do cancro do colo do útero considerou-se necessária uma colpocitologia em cada três anos nas mulheres entre os 25 e os 64 anos, inclusive (um terço das mulheres em cada ano) ${ }^{21}$ Para rastreio do cancro do cólon e do recto considerou-se necessária a realização de uma pesquisa de sangue oculto nas fezes anual nos adultos entre os 50 e os 74 anos, inclusive. ${ }^{19}$ Nos rastreios oncológicos considerou-se necessária uma consulta para colheita da história clínica, exame objectivo e pedido dos meios complementares de diagnóstico e outra para apresentação e discussão dos resultados.

O rastreio da hipertensão arterial seria feito a todos os adultos com mais de 18 anos, sem diagnóstico prévio de HTA, através de uma medição da pressão arterial a cada dois anos (metade em cada ano) ${ }^{20} \mathrm{O}$ rastreio de dislipidémia seria feito aos homens com 45 ou mais anos através de uma medição dos lípidos no sangue de cinco em cinco anos (um quinto em cada ano). ${ }^{20} \mathrm{Con}$ siderou-se necessária uma consulta para colheita da história clínica, exame objectivo e pedido dos meios complementares de diagnóstico e outra para apresentação e discussão dos resultados. O rastreio da obesidade seria feito a todos os adultos com mais de 18 anos através da determinação do índice de massa corporal a cada dois anos (metade em cada ano). ${ }^{20}$

Os dados dos utentes foram inseridos numa base de dados MySQL (Sun Microsystems). O cálculo do número de consultas necessário para realizar cada actividade durante um ano foi efectuado por um programa informático escrito em PHP (Hypertext Preprocessor)* pelos autores. Este cálculo teve por base a pirâmide etá-

*Linguagem de programação ria e a prevalência de cada situação clínica na lista. Calculou-se o número de consultas necessário para cada actividade separadamente e realizando múltiplas actividades na mesma consulta. Nos homens e mulheres não grávidas, considerou-se que a mesma consulta poderia ser utilizada para realizar várias actividades preventivas ou de seguimento de doenças crónicas, contando os utentes que realizariam múltiplas actividades apenas uma vez, excepto para o rastreio do cancro do colo do útero e vigilância da gravidez (que seriam feitos em consultas apenas com essa finalidade).

\section{RESULTADOS}

Nesta lista, 1.200 utentes tinham tido uma consulta nos cinco anos anteriores, 848 (53,4\% do total) dos quais no ano anterior. Dos restantes, 112 tinham tido a última consulta há mais de cinco anos e 266 não tinham registo de consulta prévia. Existiam 986 utentes com pelo menos um problema de saúde registado. Os dez problemas mais prevalentes eram: excesso de peso (14,2\%), abuso do tabaco (13,4\%), hipertensão sem complicações $(13,1 \%)$, alteração do metabolismo dos lípidos (12,9\%), obesidade $(10,0 \%)$, perturbação depressiva $(8,1 \%)$, diabetes não insulino-dependente $(5,0 \%)$, veias varicosas da perna $(4,8 \%)$, alteração funcional do estômago $(4,0 \%)$ e rinite alérgica $(4,0 \%)$. No ano anterior tinham sido realizadas nesta lista 3972 consultas (consultas de inter-substituição da USF não incluídas).

O Quadro I mostra o número de utentes em cada grupo e o número consultas calculado para vigilância de cada uma das situações clínicas, caso não fossem realizadas múltiplas actividades na mesma consulta. Por ano, seriam necessárias 275,5 consultas para vigiar, com uma taxa de cobertura de $80 \%$, as 250 crianças e jovens da lista; 84,8 consultas para seguir as grávidas, com uma taxa de cobertura de $80 \% ; 266,1$ consultas para vigiar os diabéticos, com uma taxa de cobertura de $85 \%$; 473,2 consultas para vigiar os hipertensos, com uma taxa de cobertura de $91 \%$; 128,5 consultas para fazer o rastreio do cancro da mama, com uma taxa de cobertura de $68 \%$; 189,1 consultas para realizar o rastreio do cancro do colo do útero, com uma taxa de cobertura de $58 \%$; 620,8 consultas para realizar o rastreio do cancro do cólon e recto, com uma taxa de cobertura de $80 \%$; 325,0 consultas para realizar o rastreio de HTA, com uma taxa de cobertura de $68 \% ; 71,8$ consultas para 


\begin{tabular}{|c|c|c|c|c|c|c|c|}
\hline \multicolumn{2}{|c|}{ Grupo } & \multirow{2}{*}{$\frac{n}{23}$} & \multirow{2}{*}{$\begin{array}{c}\text { Cobertura } \\
80 \%\end{array}$} & \multirow{2}{*}{$\begin{array}{c}\text { Consultas } \\
\text { por ano }\end{array}$} & \multirow{2}{*}{$\begin{array}{c}\text { Proporção } \\
\text { num ano }\end{array}$} & \multicolumn{2}{|c|}{ Total consultas } \\
\hline \multirow{10}{*}{ Crianças } & 0-1 ano & & & & & 110,4 & \multirow{10}{*}{275,5} \\
\hline & 1-2 anos & 18 & $80 \%$ & 3 & $100 \%$ & 43,2 & \\
\hline & 2-3 anos & 14 & $80 \%$ & 1 & $100 \%$ & 11,2 & \\
\hline & 3-4 anos & 25 & $80 \%$ & 1 & $100 \%$ & 20,0 & \\
\hline & 4-5 anos & 14 & $80 \%$ & 1 & $100 \%$ & 11,2 & \\
\hline & 5-7 anos & 52 & $80 \%$ & 1 & $50 \%$ & 20,8 & \\
\hline & 8-9 anos & 18 & $80 \%$ & 1 & $100 \%$ & 14,4 & \\
\hline & 11-14 anos & 46 & $80 \%$ & 1 & $33 \%$ & 12,3 & \\
\hline & $15-16$ anos & 18 & $80 \%$ & 1 & $100 \%$ & 14,4 & \\
\hline & 18-19 anos & 22 & $80 \%$ & 1 & $100 \%$ & 17,6 & \\
\hline \multirow[t]{2}{*}{ Grávidas } & Risco normal & 13 & $80 \%$ & 8 & $100 \%$ & 83,2 & \multirow[t]{2}{*}{84,8} \\
\hline & Alto risco & 2 & $80 \%$ & 1 & $100 \%$ & 1,6 & \\
\hline \multirow[t]{2}{*}{ Diabéticos } & Tipo 2 & 78 & $85 \%$ & 4 & $100 \%$ & 265,2 & \multirow[t]{2}{*}{266,1} \\
\hline & Outros tipos & 1 & $85 \%$ & 1 & $100 \%$ & 0,9 & \\
\hline \multicolumn{2}{|c|}{ HTA } & 260 & $91 \%$ & 2 & $100 \%$ & \multicolumn{2}{|r|}{473,2} \\
\hline \multirow{3}{*}{ Cancros } & Mama & 189 & $68 \%$ & 2 & $50 \%$ & 128,5 & \multirow{3}{*}{938,4} \\
\hline & Colo do útero & 489 & $58 \%$ & 2 & $33 \%$ & 189,1 & \\
\hline & Cólon e recto & 388 & $80 \%$ & 2 & $100 \%$ & 620,8 & \\
\hline \multirow{3}{*}{$\begin{array}{l}\text { Actividades } \\
\text { Preventivas }\end{array}$} & HTA & 956 & $68 \%$ & 1 & $50 \%$ & 325,0 & \multirow{3}{*}{810,6} \\
\hline & Dislipidémia & 264 & $68 \%$ & 2 & $20 \%$ & 71,8 & \\
\hline & Obesidade & 1217 & $68 \%$ & 1 & $50 \%$ & 413,8 & \\
\hline \multicolumn{2}{|c|}{ Total } & & & & & \multicolumn{2}{|r|}{2848,5} \\
\hline
\end{tabular}

realizar o rastreio de dislipidémia, com uma taxa de cobertura de $68 \%$; e 413,8 consultas para realizar o rastreio de obesidade, com uma taxa de cobertura de $68 \%$. No total, seriam necessárias 2848,5 consultas para levar a cabo as actividades preventivas e de vigilância estudadas.

Se se considerar a possibilidade de serem realizadas múltiplas actividades preventivas na mesma consulta, o número de consultas necessário é menor. A Figura 1 mostra o número de utentes englobado em cada actividade preventiva, considerando a possibilidade de actividades múltiplas. Assim, o número de utentes-alvo permanece constante na saúde infantil e juvenil (uma vez que as restantes actividades só se aplicam a adul- tos), na vigilância das grávidas e no rastreio do cancro do colo do útero (que se definiram no desenho do estudo não poderem ser realizadas em conjunto com outras actividades) e no seguimento de doentes diabéticos (tomados como população de referência por terem maior número de consultas de vigilância por ano).

Dos utentes hipertensos, existiam 62 que eram ao mesmo tempo diabéticos. Considerando uma taxa de cobertura dos diabéticos de 85\%, 52,7 utentes já teriam tido quatro consultas naquele ano. Ficariam 207,3 hipertensos por vigiar, necessitando de 377,3 consultas.

Nas mulheres entre os 50 e os 69 anos, existiam 20 diabéticas e 61 com HTA e sem diabetes, cujo rastreio do cancro da mama poderia ser feito nas consultas de 


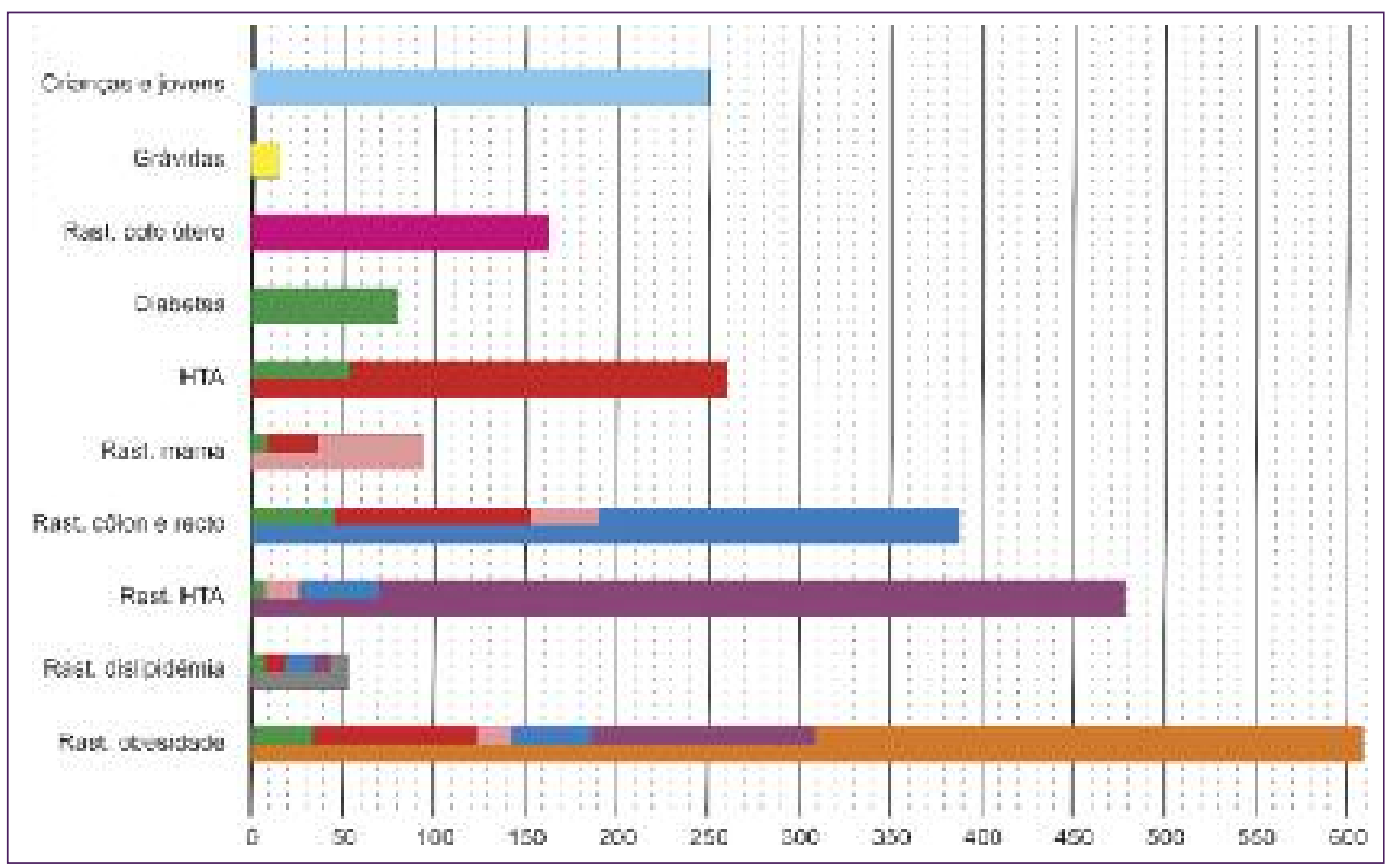

Figura 1. Número de utentes englobado em cada actividade preventiva ou de vigilância em cada ano, assinalando as sobreposições. As barras mais finas indicam a proporção de utentes já vigiada naquele ano no âmbito de outra actividade (ver explicação detalhada no texto). HTA - hipertensão arterial.

diabetes e hipertensão. Tendo em conta as taxas de cobertura da vigilância de diabéticos e hipertensos, ficariam por fazer o rastreio do cancro da mama $116,5 \mathrm{mu}-$ lheres (58,3 por ano), necessitando de 79,2 consultas por ano para atingir uma taxa de cobertura de $68 \%$.

Nos adultos em idade de fazer o rastreio do cancro do cólon e recto, existiam 53 diabéticos, 118 hipertensos sem diabetes e 108 (54 em cada ano) mulheres em idade de realizar o rastreio do cancro da mama e sem diabetes nem HTA. Assim, 198,9 pessoas necessitariam de realizar o rastreio do cancro colo-rectal fora do contexto de outra actividade preventiva ou de vigilância, correspondendo a 318,2 consultas por ano.

Existiam 1217 pessoas com 19 ou mais anos, 260 dos quais hipertensos (pelo que sem necessidade de fazer rastreio da HTA). Dos restantes 957, 17 eram diabéticos sem HTA, 108 eram mulheres em idade de fazer o rastreio do cancro da mama e sem diabetes nem HTA (54 em cada ano) e 109 deveriam fazer o rastreio do cancro do cólon e do recto e não tinham diabetes, HTA ou rastreio do cancro da mama. Deste modo, 818,6 pessoas deveriam vir à consulta exclusivamente para fazer o rastreio da HTA (409,3 em cada ano), necessitando de 278,3 consultas para atingir a taxa de cobertura de $68 \%$.

Existiam 264 homens em idade de fazer o rastreio da dislipidémia. Destes, 41 eram diabéticos, 61 hipertensos sem diabetes, 97 deveriam fazer o rastreio do cancro do cólon e recto e não tinham diabetes ou HTA e 65 o rastreio da HTA (32,5 em cada ano) e não tinham diabetes ou o rastreio do cancro do cólon e recto. Assim, 73,9 homens deveriam fazer o rastreio da dislipidémia (14,8 em cada ano), necessitando de 20,1 consultas.

Existiam 1217 pessoas que deveriam fazer o rastreio da obesidade. Destes, 79 eram diabéticos, 198 hipertensos sem diabetes, 108 deveriam fazer o rastreio do cancro da mama (54 em cada ano) e não tinham diabetes ou HTA, 109 deveriam fazer o rastreio do cancro do cólon e recto e não tinham diabetes, HTA ou rastreio do cancro da mama, 723 deveriam fazer o rastreio da HTA (361,5 em cada ano) e não tinham diabetes, HTA, rastreio do cancro da mama ou do cancro do cólon e recto e nenhum utente ainda não observado por outro 


\begin{tabular}{|c|c|c|c|c|c|c|c|}
\hline \multicolumn{2}{|c|}{ Grupo } & \multirow{2}{*}{$\begin{array}{r}n \\
23\end{array}$} & \multirow{2}{*}{$\begin{array}{c}\text { Cobertura } \\
80 \%\end{array}$} & \multirow{2}{*}{$\begin{array}{c}\text { Consultas } \\
\text { por ano }\end{array}$} & \multirow{2}{*}{$\begin{array}{c}\begin{array}{c}\text { Proporção } \\
\text { num ano }\end{array} \\
100 \%\end{array}$} & \multicolumn{2}{|c|}{ Total consultas } \\
\hline \multirow{10}{*}{ Crianças } & $0-1$ ano & & & & & 110,4 & \multirow{10}{*}{275,5} \\
\hline & 1-2 anos & 18 & $80 \%$ & 3 & $100 \%$ & 43,2 & \\
\hline & 2-3 anos & 14 & $80 \%$ & 1 & $100 \%$ & 11,2 & \\
\hline & 3-4 anos & 25 & $80 \%$ & 1 & $100 \%$ & 20,0 & \\
\hline & 4-5 anos & 14 & $80 \%$ & 1 & $100 \%$ & 11,2 & \\
\hline & 5-7 anos & 52 & $80 \%$ & 1 & $50 \%$ & 20,8 & \\
\hline & 8-9 anos & 18 & $80 \%$ & 1 & $100 \%$ & 14,4 & \\
\hline & 11-14 anos & 46 & $80 \%$ & 1 & $33 \%$ & 12,3 & \\
\hline & 15-16 anos & 18 & $80 \%$ & 1 & $100 \%$ & 14,4 & \\
\hline & 18-19 anos & 22 & $80 \%$ & 1 & $100 \%$ & 17,6 & \\
\hline \multirow[t]{2}{*}{ Grávidas } & Risco normal & 13 & $80 \%$ & 8 & $100 \%$ & 83,2 & \multirow[t]{2}{*}{84,8} \\
\hline & Alto risco & 2 & $80 \%$ & 1 & $100 \%$ & 1,6 & \\
\hline \multirow[t]{2}{*}{ Diabéticos } & Tipo 2 & 78 & $85 \%$ & 4 & $100 \%$ & 265,2 & \multirow[t]{2}{*}{266,1} \\
\hline & Outros tipos & 1 & $85 \%$ & 1 & $100 \%$ & 0,9 & \\
\hline \multicolumn{2}{|c|}{ HTA } & 207,3 & $91 \%$ & 2 & $100 \%$ & \multicolumn{2}{|r|}{377,3} \\
\hline \multirow{3}{*}{ Cancros } & Mama & 116,5 & $68 \%$ & 2 & $50 \%$ & 79,2 & \multirow{3}{*}{586,5} \\
\hline & Colo do útero & 489 & $58 \%$ & 2 & $33 \%$ & 189,1 & \\
\hline & Cólon e recto & 198,9 & $80 \%$ & 2 & $100 \%$ & 318,2 & \\
\hline \multirow{3}{*}{$\begin{array}{l}\text { Actividades } \\
\text { Preventivas }\end{array}$} & HTA & 818,6 & $68 \%$ & 1 & $50 \%$ & 278,3 & \multirow{3}{*}{418,8} \\
\hline & Dislipidémia & 73,9 & $68 \%$ & 2 & $20 \%$ & 20,1 & \\
\hline & Obesidade & 354,1 & $68 \%$ & 1 & $50 \%$ & 120,4 & \\
\hline \multicolumn{2}{|c|}{ Total } & & & & & \multicolumn{2}{|r|}{2008,9} \\
\hline
\end{tabular}

motivo tinha indicação para fazer o rastreio da dislipidémia. Assim, 354,1 pessoas deveriam fazer o rastreio da obesidade (177,1 em cada ano), necessitando de 120,4 consultas.

O Quadro II resume o número de utentes alvo de cada rastreio e o número de consultas necessário considerando a realização de múltiplas actividades preventivas. No total, seriam necessárias 2008,9 consultas por ano (50,6\% do total realizado no ano anterior). A Figura 2 ilustra a proporção de consultas necessária para completar cada actividade face ao total de consultas do ano anterior, considerando a existência de sobreposição sempre que na mesma consulta fosse realizada mais do que uma actividade.

\section{DISCUSSÃO}

Nesta estimativa, as actividades preventivas e de vigilância consideradas ocupariam o equivalente a metade das consultas realizadas no ano anterior a utentes desta lista. O médico de família teria de distribuir o tempo remanescente pelos cuidados às pessoas com doença aguda, com outras doenças crónicas (doenças psiquiátricas, endócrinas, do aparelho musculo-esquelético, do sistema digestivo, etc.), prevenção de factores de risco não considerados (como o tabagismo e o sedentarismo), educação para a saúde, consultas no domicílio, actividades burocráticas em pessoas sem doença, contactos indirectos e outras actividades.

Estes resultados estão de acordo com outros traba- 


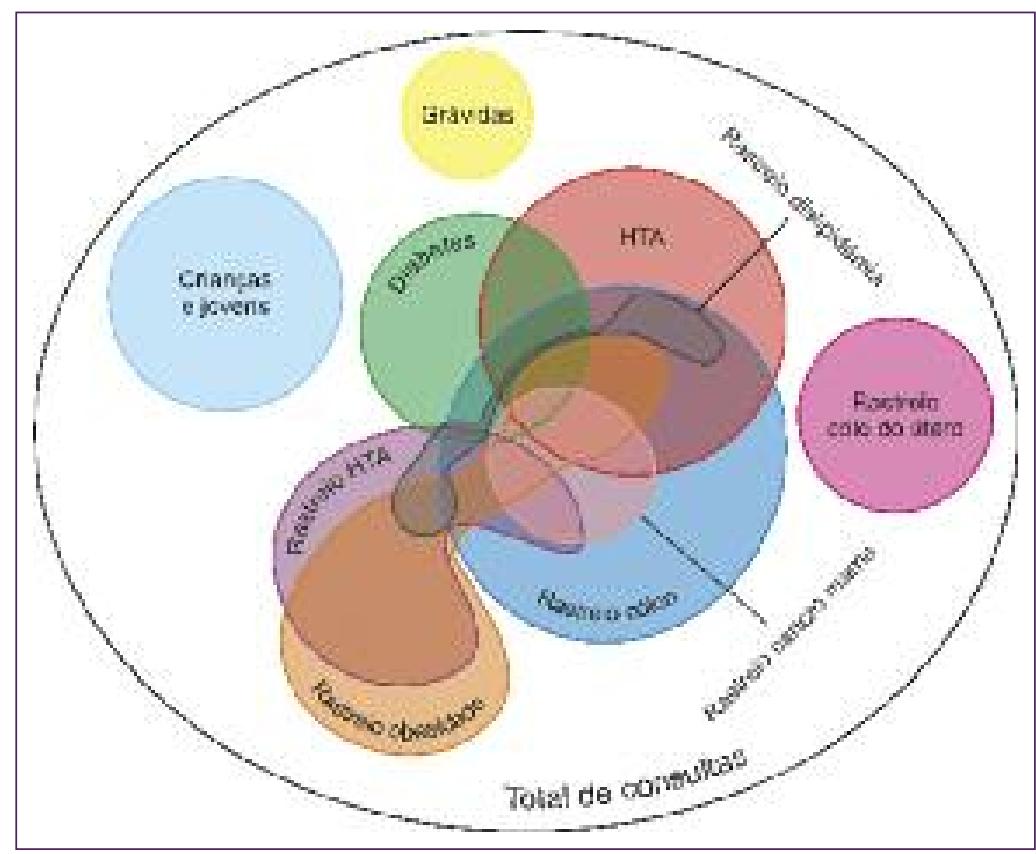

Figura 2. Representação proporcional das necessidades de consultas para cada actividade, incluindo sobreposições quando múltiplas actividades poderiam ser realizadas na mesma consulta. A área de cada actividade é directamente proporcional ao número de consultas necessário; as áreas de sobreposição são aproximadas.

duzindo a dimensão da sua lista de utentes) ou parte das actividades preventivas e de vigilância poderiam passar a ser realizadas por outros profissionais de saúde, libertando o médico para as situações de doença. Porém, todas estas alternativas implicariam maior dispêndio de recursos económicos e humanos.

Esta observação põe em evidência a necessidade de avaliar o impacto sistémico das recomendações feitas aos médicos de família, enquadrando-as no conjunto de actividades realizadas por estes profissionais. O imperativo de considerar a viabilidade, sustentabilidade e impacto real sobre a saúde da população da aplicação de normas de orientação clínica em larga escala em cuidados primários tem sido amplamente demonstrado na área das doenças cardiovasculares. ${ }^{23-26}$

Os resultados deste trabalho devem entender-se como exploratórios. Procurou-se quantificar um problema que

lhos que mostram que o tempo necessário para seguir as recomendações relacionadas com actividades preventivas e vigilância de doenças crónicas é superior ao que os médicos de família podem acomodar. ${ }^{13,22}$ Não conhecemos nenhum trabalho feito em Portugal com uma metodologia idêntica à que utilizámos. O único com algumas semelhanças, realizado por Fernandes e colaboradores em 1994, debruça-se sobretudo sobre a organização do horário do médico de família. Neste trabalho assume-se que seriam necessárias 3.686 consultas para uma lista de 1.024 utentes, o que equivaleria a 5.713 consultas numa lista de 1.587 utentes (mais $43,8 \%$ que o total realizado no ano anterior). Assim, ambos os trabalhos sugerem que também em Portugal será difícil ao médico de família conseguir realizar todas estas actividades no tempo limitado de que dispõe.

Não sendo possível executar todas as actividades, seria útil definir aquelas que são prioritárias ou que podem traduzir-se em maiores ganhos em saúde, de acordo com a melhor evidência disponível. Em alternativa, deveria ser atribuído ao médico de família mais tempo para a sua realização (alargando o seu horário ou remuitos médicos de família sentem na sua prática clínica através de uma metodologia imperfeita. Para realizar este trabalho definiu-se um conjunto de premissas teóricas acerca do que é ideal e que sustentaram os cálculos efectuados. Porém, nem todas estas premissas serão verificáveis na prática e é essa a principal limitação à validade externa dos resultados.

Utilizaram-se recomendações e normas de orientação clínica que são habitualmente seguidas pelos autores, mas que poderão não ser aceites por todos os médicos. Já as regras de avaliação dos indicadores de desempenho são iguais em todas as USFs, mas o mesmo não se passa com as taxas de cobertura contratualizadas. Em USFs que contratualizem taxas de cobertura mais elevadas, o número de consultas necessário para realizar estas actividades será maior.

Algumas das actividades preventivas e de vigilância enumeradas poderiam ser realizadas no âmbito de consultas realizadas por outro motivo, não necessitando de uma consulta própria. Por exemplo, numa consulta de uma mulher de 52 anos que recorre ao seu médico por lombalgia, podem ser feitos os rastreios do cancro da 
mama, do cancro do cólon e recto, da hipertensão arterial e da obesidade. Porém, se essa utente, para além da lombalgia, apresentar mais dois ou três problemas de saúde nessa consulta (por exemplo, preocupação por um nevo cutâneo e insónia), já será bastante mais difícil ao médico conseguir fazer todos os rastreios indicados no tempo limitado da consulta. Da mesma forma, quando definimos que cada utente poderia fazer todas actividades preventivas e de vigilância na mesma consulta, poderemos ter subestimado as necessidades reais. Uma estimativa mais precisa poderia ser conseguida se, em vez de calcular o número de consultas, fosse calculado o tempo necessário para cada actividade (estimando a partir daí o número de consultas correspondente). Contudo, em muitas actividades é difícil estimar o número de minutos necessário para a sua realização e a variabilidade entre consultas e entre médicos será seguramente grande. Existem trabalhos semelhantes ao nosso, que utilizam um cálculo baseado no tempo necessário para executar cada actividade. ${ }^{13,22}$ Contudo, é difícil transportar esta informação da realidade dos Estados Unidos da América para Portugal, onde não existem physician assistants, nurse practitioners e outros profissionais a auxiliar os médicos. Para que possa ser efectuado um cálculo baseado no tempo necessário para as actividades preventivas e de vigilância de doenças crónicas será primeiro necessário realizar trabalhos que façam essa medição no contexto dos centros de saúde portugueses.

Há ainda que considerar que a estimativa poderá ser demasiado conservadora, ao supor que não são realizadas consultas de saúde infantil fora das idades-chave recomendadas, que os hipertensos são vigiados apenas duas vezes por ano, que todas as mulheres em idade de fazer o rastreio do cancro do colo do útero apenas necessitam de fazer citologias com um intervalo de três anos, que as mulheres em idade de fazer mamografia o fazem apenas de dois em dois anos e que o rastreio da dislipidémia é feito apenas aos homens (excluindo as mulheres com factores de risco cardiovascular) e só de cinco em cinco anos.

Não foram também considerados os utentes já com diagnóstico de excesso de peso ou obesidade e aqueles já com o diagnóstico de dislipidémia e que não necessitariam de fazer o rastreio destes factores de risco. Porém, estas pessoas necessitarão de consultas de segui- mento que provavelmente serão mais frequentes do que o definido para o rastreio, pelo que as suas necessidades estão subestimadas.

Por último, os valores apresentados reflectem a composição da lista de utentes estudada em termos de pirâmide etária e prevalência dos problemas estudados. Além disso, existe uma porção significativa dos inscritos nesta lista que nunca utilizaram a consulta do médico de família. Possivelmente, algumas dessas pessoas poderão ter hipertensão ou diabetes ou estar grávidas sem o conhecimento do médico. Finalmente, a lista estudada tem dimensões que estão de acordo com os cerca de 1.500 utentes exarados na lei, mas reconhecemos que a maioria das listas (particularmente em USFs) têm dimensões consideravelmente superiores, o que agudiza o problema da incapacidade de prestar cuidados globais de saúde. Assim, é necessário cuidado ao extrapolar estes resultados para as listas de outros médicos.

Em conclusão, a realização das actividades preventivas e o cumprimento de indicadores de desempenho exigem um dispêndio considerável de tempo e poderão limitar o tempo disponível para médico de família cuidar de pessoas doentes. Antes de serem atribuídas mais tarefas aos médicos de família, será necessário estudar o impacto de cada uma em termos de recursos a despender para a sua execução. Dado que cada nova tarefa introduzida vai interferir com o cumprimento de outras, impossibilitando a prestação de cuidados globais de saúde, característica nuclear da especialidade de Medicina Geral e Familiar, a implementação de novos procedimentos deve ter em atenção os recursos existentes e verificar se, realmente, têm que ser feitos pelo médico de família ou se podem ser delegados noutros profissionais de saúde.

\section{REFERÊNCIAS BIBLIOGRÁFICAS}

1. Hespanhol AP, Couto L, Martins C, Viana M. Educação para a saúde e prevenção na consulta de medicina geral e familiar (I). Rev Port Clin Geral 2009 Mar-Abr; 25 (2):236-41.

2. Starfield B, Hyde J, Gérvas J, Heath I. The concept of prevention: a good idea gone astray? J Epidemiol Community Health 2008 Jul; 62 (7):5803.

3. Gérvas J, Starfield B, Heath I. Is clinical prevention better than cure? Lancet 2008 Dec 6; 372 (9654): 1997-9.

4. Moynihan R, Heath I, Henry D. Selling sickness: the pharmaceutical industry and disease mongering. BMJ 2002 Apr 13; 324 (7342): 886-91.

5. Heath I. In defence of a National Sickness Service. BMJ 2007 Jan 6; 334 
(7358): 19.

6. Weingarten $M$, Matalon A. The ethics of basing community prevention in general practice. J Med Ethics 2010 Mar; 36 (3): 138-41.

7. Contratualização com as Unidades de Saúde Familiares para 2007. Agências de Contratualização dos Serviços de Saúde. Disponível em: http://mcsp.lvengine.com/Imgs/content/page_46/Contratualizacao\%20-\%202007.pdf [acedido a 02/10/2009].

8. Unidades de Saúde Familiar - Metodologia de Contratualização. Missão para os Cuidados de Saúde Primários. Janeiro 2009. Disponível em: http://www.mcsp.min-saude.pt/Imgs/content/page_46/Contratualizacao_USF_2009_v_1_3.pdf [acedido a 02/10/2009].

9. Normas para cálculo das remunerações dos profissionais das USF Modelo B. Missão para os Cuidados de Saúde Primários. Abril 2008. Disponível

em: http://mcsp.lvengine.com/Imgs/content/page_46/MCSP_Remuneracoes_ModB.pdf [acedido a 02/10/2009].

10. Campbell SM, Reeves D, Kontopantelis E, Sibbald B, Roland M. Effects of pay for performance on the quality of primary care in England. $N$ Engl J Med 2009 Jul 23; 361 (4): 368-78.

11. Adair CE, Simpson E, Casebeer AL, Birdsell JM, Hayden KA, Lewis S. Performance measurement in healthcare: part II - state of the science findings by stage of the performance measurement process. Healthc Policy 2006 Jul; 2 (1): 56-78.

12. Heath I, Hippisley-Cox J, Smeeth L. Measuring performance and missing the point? BMJ 2007 Nov 24; 335 (7629): 1075-6.

13. Yarnall KS, Pollak KI, Østbye T, Krause KM, Michener JL. Primary care: is there enough time for prevention? Am J Public Health 2003 Apr; 93 (4): 635-41.

14. Fernandes A, Arnaud F, Carvalho L, Monsanto L, Almeida M. Programação de actividades numa lista de utentes de que se conhece a pirâmide etária. Rev Port Clin Geral 1994;12: 303-9.

15. Comissão Internacional de Classificações daWONCA, Classificação Internacional de Cuidados Primários - ICPC-2. 2ª ed. Lisboa: Associação Portuguesa dos Médicos de Clínica Geral; 1999.

16. Saúde Infantil e Juvenil - Programa-Tipo de Actuação. $2^{a}$ ed. Lisboa: Direcção-Geral da Saúde; 2005.

17. Vigilância Pré-Natal e Revisão do Puerpério. $2^{\mathrm{a}}$ ed. Lisboa: DirecçãoGeral da Saúde; 1993.

18. Circular Normativa $n^{\circ}$ 2/DGCG - Diagnóstico, Tratamento e Controlo da Hipertensão Arterial. Lisboa: Direcção-Geral da Saúde; 2004.

19. United States Preventive Services Task Force. Guide to Clinical Preventive Services. Agency for Healthcare Research and Quality. 2009.
Disponível em: http://www.ahrq.gov/clinic/pocketgd.htm [acedido a 02/10/2009].

20. The Royal Australian College of General Practitioners 'Red Book' Taskforce. Guidelines for preventive activities in general practice. 2nd ed. Melbourne: The Royal Australian College of General Practitioners; 2009.

21. Rosendo I, Ribeiro E,Almada-Lobo F, Martins C. Recomendações do Núcleo de Actividades Preventivas da APMCG - Cancro do colo do útero. Setembro 2008. Disponível em: http://www.apmcg.pt/files/54/documentos/20081104113222421666.pdf [acedido a 02/10/2009].

22. Østbye T, Yarnall KS, Krause KM, Pollak KI, Gradison M, Michener JL. Is there time for management of patients with chronic diseases in primary care? Ann Fam Med 2005 May-Jun; 3 (3): 209-14.

23. Petursson H, Getz L, Sigurdsson JA, Hetlevik I. Current European guidelines for management of arterial hypertension: are they adequate for use in primary care? Modelling study based on the Norwegian HUNT 2 population. BMC Fam Pract 2009 Oct 30;10:70.

24. Hartz I, Njølstad I, Eggen AE. Does implementation of the European guidelines based on the SCORE model double the number of Norwegian adults who need cardiovascular drugs for primary prevention? The Tromsø study 2001. Eur Heart J 2005 Dec; 26 (24): 2673-80.

25. Getz L, Kirkengen AL, Hetlevik I, Romundstad S, Sigurdsson JA. Ethical dilemmas arising from implementation of the European guidelines on cardiovascular disease prevention in clinical practice. A descriptive epidemiological study. Scand J Prim Health Care 2004 Dec; 22 (4): 202-8.

26. Thomson R, Greenaway J, Chinn DJ, Wood R, Rodgers H. The impact of implementing national hypertension guidelines on prevalence and workload in primary care: a population-based survey of older people. J Hum Hypertens 2005 Sep; 19 (9): 683-9.

\section{CONFLITOS DE INTERESSE}

Daniel Pinto é editor da RPCG e não esteve envolvido no processo de revisão editorial do presente artigo. Os restantes autores declararam não possuir conflitos de interesses.

\section{ENDEREÇO PARA CORRESPONDÊNCIA}

Daniel Pinto

Email: danieljllpinto@gmail.com

Morada: Rua da Fonte, 7 - Tornada

2500-315 Caldas da Rainha

Recebido em 30/01/2010

Aceite para publicação em 13/09/2010 


\section{ABSTRACT}

\section{PREVENTIVE ACTIVITIES AND PERFORMANCE INDICATORS - HOW MUCH TIME IS LEFT?}

Objectives: To determine the number of office visits needed per year to perform selected preventive procedures and, in patients with diabetes or hypertension, meet performance indicators related with follow-up.

Methods: Descriptive study based on the list of patients of a family doctor (1587 patients). We considered the follow-up of children and teenagers, pregnant women, patients with diabetes or hypertension, and screening for breast, cervical and colon cancers, hypertension, dyslipidemia and obesity. Appropriate follow-up for each situation was defined according to clinical practice guidelines, the coverage rate contractually agreed by the clinic and performance indicators defined by the Portuguese Mission for Primary Health Care. We considered the performance of each activity separately or of multiple preventive and followup activities in the same visit.

Results: If they were held separately, we would need 2848.5 office visits to carry out the defined preventive activities and follow-up of patients with diabetes or hypertension. If multiple activities were performed in the same visit, we would require 2008.9 consultations per year ( $50.6 \%$ of the total in the previous year).

Conclusions: Implementation of preventive activities and compliance with performance indicators require a considerable amount of time. Before assigning more tasks to family physicians, the weight of each on existing resources should be assessed.

Keywords: Family Practice; Health Resources; Pay for Performance; Primary Prevention.

\section{capa}

\section{SARA CAMPOS}

Sara Campos é interna do $3 .^{\circ}$ ano de MGF no Centro de Saúde de Mangualde. Tem 29 anos, é auto-didacta desde os 17 anos, pinta em acrílico e nunca realizou nenhuma exposição

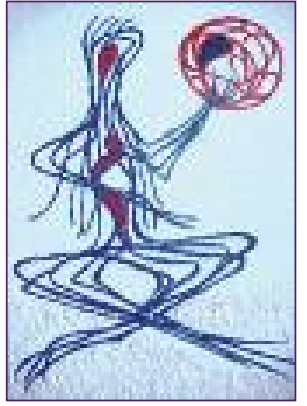

Poder

Sara Campos

Pintura sobre acrílico 